\title{
Robotique éducative et constitution de communs de la connaissance dans les FabLabs : un enjeu fondamental pour le développement
}

Educational robotics and building knowledge commons in FabLabs: a

fundamental challenge for development

La robótica educativa y la constitución del patrimonio de conocimientos en los

FabLabs: un desafío fundamental para el desarrollo

Anne Lehmans, Vincent Liquète et Mohamed Coulibaly

\section{(2) OpenEdition}

Journals

Édition électronique

URL : http://journals.openedition.org/ctd/3093

DOI : $10.4000 /$ ctd.3093

ISSN : 2491-1437

Éditeur

Chaire Unesco Pratiques émergentes en technologies et communication pour le développement

Édition imprimée

ISBN : 2491-1437

Référence électronique

Anne Lehmans, Vincent Liquète et Mohamed Coulibaly, « Robotique éducative et constitution de communs de la connaissance dans les FabLabs : un enjeu fondamental pour le développement », Communication, technologies et développement [En ligne], 8 | 2020, mis en ligne le 30 juin 2020, consulté le 29 mars 2021. URL : http://journals.openedition.org/ctd/3093 ; DOI : https://doi.org/ $10.4000 /$ ctd.3093

Ce document a été généré automatiquement le 29 mars 2021.

Communication, technologies et développement 


\title{
Robotique éducative et constitution de communs de la connaissance dans les FabLabs : un enjeu fondamental pour le développement
}

\author{
Educational robotics and building knowledge commons in FabLabs : a \\ fundamental challenge for development \\ La robótica educativa y la constitución del patrimonio de conocimientos en los \\ FabLabs: un desafío fundamental para el desarrollo
}

Anne Lehmans, Vincent Liquète et Mohamed Coulibaly

1 La perception de l'espace public a évolué ces dernières années vers une revendication diffuse d'un droit universel d'accès à l'information, aux savoirs et aux compétences nécessaires au développement économique et social et à la participation aux débats publics, notamment autour du numérique et des promesses de l'intelligence artificielle, que l'on peut considérer comme une part des « communs de la connaissance » (Ostrom, 2015). Dans le socle commun de la société de la connaissance, on trouve les bases d'une culture numérique constituée autour de la machine, du langage, de l'algorithme et de l'information, à partir des trois dimensions que sont les façons de penser, les objets et les interactions sociales à l'intérieur des réseaux. Jeannette Wing (2006), parle de pensée computationnelle, mettant en œuvre plusieurs niveaux d'abstraction axés sur la résolution de problèmes, la capacité à nommer les objets, dans le contexte du monde réel et des objets qui le composent. Bruno Bachimont (2015) parle de raison computationnelle, dans laquelle le programme, le réseau et la couche caractérisent une technologie de l'intellect et une littératie propres à l'écriture numérique (Goody, 1979). La robotique éducative est amenée à jouer un rôle particulier et renouvelé dans cette littératie, depuis quelques années, interrogeant le lien entre la forme de l'objet et ses potentialités d'apprentissage, se posant comme "science et pratique de l'incarnation " (Kaplan, Oudeyer, 2008). Aux dimensions socio-économique de production, et psychologique de projection (Baudrillard, 1968) et d'empathie (Tisseron, 2015) 
attribuées au robot comme objet, les roboticiens ajoutent une dimension cognitive de modélisation des apprentissages, et les pédagogues une dimension éducative de motivation des apprentissages de l'écriture numérique et plus précisément de la programmation. La robotique éducative semble apporter un élément de réponse à l'appel de Gilbert Simondon (2009) à mettre en phase la culture avec la réalité, et à considérer l'objet technique comme un objet-réseau.

2 Cette revendication passe notamment par l'acquisition de compétences renouvelées, avec la capacité à construire des objets dans une démarche collaborative et à l'aide de techniques et d'outils mis à disposition du public dans des espaces ouverts et partagés. Le concept de FabLab y répond. Créé par Neil Gershenfeld, professeur au MIT, le «laboratoire de fabrication » est un lieu ouvert au public où sont mis à disposition toutes sortes d'outils, avec des données stockées sur des plateformes collaboratives, en vue de concevoir et de réaliser des objets. Le travail qui s'y mène dans un collectif à géométrie variable, ne porte pas seulement sur les aspects matériels de la fabrication d'objets, mais aussi sur les développements informatiques nécessaires pour les faire fonctionner, notamment autour de la robotique, et sur les dimensions sociales de la création. Ces développements sont eux-mêmes partagés sur des plateformes collaboratives.

3 Dans l'histoire de la pensée computationnelle aussi bien que des évolutions techniques du numérique et de l'internet, le FabLab s'est développé sur un terreau militant, promouvant une culture scientifique et technique partagée (Stallman, 2017). La robotique éducative dans les FabLabs en fait aussi des lieux de développement de stratégies pédagogiques et éducatives qui associent des acteurs différents, en permettant des interactions autour des compétences en jeu. C'est à ce titre qu'ils font l'objet d'un projet de recherche, qui tente d'analyser, dans une approche longitudinale, les effets réels de l'usage d'objets tangibles (les robots notamment), en comparant les contextes scolaires et non scolaires des Fablabs, espaces de co-création dans la réflexion collective et la production collaborative. Autour de ces usages, un réseau de recherche et de formation s'est constitué sur la base d'échanges de données, d'expériences et de ressources pédagogiques, dans des espaces collaboratifs ouverts. Les FabLabs analysés dans le projet sont liés au contexte scolaire par leur appartenance institutionnelle (université de Bordeaux) ou par leur vocation culturelle. A l'occasion de cette recherche, qui implique de nombreux chercheurs notamment en informatique, le projet est né de créer un Fablab correspondant dans l'université de Ségou au Mali, en s'appuyant sur le développement d'une intelligence non seulement artificielle mais surtout collaborative, qui ne nécessite pas de moyens matériels ou techniques importants, mais plutôt la construction d'une communauté de pratique à distance.

4 La question que nous proposons d'analyser dans le cadre de ce projet et dans la perspective du colloque est celle de l'effet de cet espace très particulier qu'est le FabLab sur la dynamique et le partage des apprentissages. L'hypothèse est que pour certains élèves, enfants, jeunes ou moins jeunes adultes, l'école constitue un cadre d'échec, ou dans certains pays une voie impossible pour la formation, et le FabLab une possibilité de retrouver confiance, envie, interactions sociales pour apprendre. Dans une démarche qualitative basée sur l'observation participative et des entretiens menés auprès des participants et des responsables des FabLabs, nous tenterons de faire ressortir, en nous centrant sur le cas du Mali, et en examinant l'émergence d'un projet de construction d'un FabLab, les conditions qui nous semblent indispensables pour que 
le Fablab devienne un lieu d'apprentissage de la culture numérique d'une part, un espace de développement d'une communauté de pratique et d'une culture partagée, d'autre part.

\section{Les Fablabs dans la philosophie des communs de la connaissance}

\section{La connaissance en communs}

\section{Une approche des communs}

5 La culture technique est aujourd'hui nécessaire pour que tout individu puisse agir avec et sur son environnement, gage de sa liberté et de son indépendance dans le contexte de l'industrialisation des connaissances par le numérique. À la fin des années 1990, les communautés des défenseurs du logiciel libre, le plus souvent militants, se développent sur la base du bénévolat et du volontariat, à l'instar de la communauté Wikipédienne. Ces groupes souhaitent démocratiser la culture informatique entendue non pas seulement comme un ensemble de compétences procédurales liées aux ordinateurs et à la programmation, mais aussi comme la capacité à comprendre la place du numérique dans les rapports sociaux, à résister à l'informatique d'entreprise, propriétaire, et à préserver des "communs de la connaissance" (Stallman, 2017), des espaces qui échappent à l'appropriation privée. Ces espaces connus sous les noms Fablabs, hackerspace ou makerspace (Lhoste \& Barbier, 2016), suivent le mouvement " hacker " qui s'inscrit dans la culture open source, du faire soi-même (Do It Yourself) et du faire avec les autres (Do it With Others) (Suire, 2016). Ils proposent des outils et savoir-faire répondant à la question «comment fabriquer à peu près n'importe quoi » (How to make (almost) anything)) ». Ils regroupent des personnes issues milieux sociaux, âges et métiers très variés, le souci de fabriquer étant ce qui les rassemble, dans un espace de rencontre et de création collaborative. Tous types d'objets en sortent, objets artistiques, objets de remplacement, prothèses, orthèses, outils. Ils s'appuient sur des machines de fabrication numérique et des réseaux qui permettent de s'échanger des fichiers dans le monde entier. Ce sont également des lieux de développement de stratégies pédagogiques et éducatives qui associent des acteurs différents, en permettant des interactions autour des compétences en jeu.

\section{Une approche collaborative}

6 Le courant pragmatiste représenté notamment par John Dewey considère l'élève comme un individu capable d'agir dans la société et non l'instrument d'un système qui le dépasse. Cette activité, indissociable d'un engagement, suppose que l'apprenant soit considéré comme sujet agissant, acteur de son propre apprentissage et non réceptacle d'un savoir accumulé. La connaissance acquise par l'expérience est celle d'un être socialement situé dans le monde, qui interagit nécessairement dans des groupes et avec des outils qui lui permettent d'appréhender ce monde. L'usage d'un dispositif numérique spécifique incluant un espace, des outils et des relations sociales est supposé apporter un cadre d'engagement dans les apprentissages. Célestin Freinet insiste sur l'éducation du travail et la "nécessité organique d'user le potentiel de vie à une activité tout à la fois individuelle et sociale, qui ait un but parfaitement compris, et présentant 
une grande amplitude de réactions (...)" en valorisant le "sentiment de puissance" (Freinet, p.157). L'un des défis de la recherche est celui de déterminer la place respective de la situation de projet, de l'espace technique et des interactions sociales dans l'étayage de la motivation et des apprentissages. Cet étayage repose sur le développement des compétences psychosociales nécessaires au travail collaboratif dans des équipes diversifiées, de la créativité, d'une démarche entrepreneuriale, une éducation à une démarche écocitoyenne, des compétences numériques et techniques avec une concrétisation sous forme d'un prototype tangible, l'incitation à la mise en oeuvre d'une démarche transversale et complexe.

7 Il est inutile de convoquer ici l'innovation, concept souvent illusoire ou utopique. Le FabLab, pas plus que l'utilisation de la robotique, ne constituent une innovation, en ce qu'ils mobilisent des compétences techniques dans une démarche de projet, ou plus précisément un apprentissage basé sur la conception (design-based learning) qui n'a rien de nouveau. André Tricot (2017), qui critique la rhétorique de l'innovation, souligne que tous les dispositifs de travail en projet autour du "faire " ne sont pas efficaces sur les apprentissages qui requièrent attention et engagement, outre la mobilisation de ressources cognitives importantes. Et que les apprentissages en jeu sont spécifiques : dans le FabLab, on ne peut pas tout apprendre, mais seulement des connaissances techniques, qui, certes, peuvent servir de levier social à l'envie d'apprendre. Certaines compétences liées à la manipulation de l'information et des ressources documentaires sont également en jeu, à travers la lecture ou la création de documentation technique, ou les projets qui associent la fabrication d'un objet avec des ressources culturelles nécessitant des recherches d'informations ou de documents. Dans le FabLab, outre les objectifs, les outils, le réseau social, c'est l'organisation spatiale des apprentissages qui est mise en évidence, ainsi que la création d'un système d'information qui permet à de la documentation technique, notamment, de circuler.

\section{La pédagogie en commun : le travail autonome}

\section{La question du travail et de la production autonomes}

8 Le travail autonome est au cœur de la réflexion pédagogique, comme "moyen de développement personnel" et comme finalité éducative (Freinet, 1994: 46). Cette autonomie a une dimension politique si l'on considère les finalités de l'éducation, qui doit construire des individus capables de s'inscrire de façon active et critique dans l'espace public. Pour Freinet, l'apprentissage relève de l'expérience tâtonnée empirique puis méthodique et scientifique, outils et langage étant intimement liés pour construire l'autonomie sur l'expérience. La question de l'autonomie est souvent assimilée à celle de l'individualisation des apprentissages. Or, travailler en autonomie s'apprend et passe par une prise en charge constante en vue d'atteindre une part de distance vis-à-vis de ses propres acquis et apprentissages (Liquète, Maury, 2007). Le travail autonome nécessite dès lors des formes d'anticipation et des scénarisations pensées et mesurées par l'équipe pédagogique afin que l'apprenant ne perde pas le fil des objectifs à atteindre tout en conservant une part d'initiative personnelle face à la tâche. L'une des plus grandes difficultés pour l'enseignant est de suivre suffisamment précisément les groupes afin d'identifier la part de travail et de production de chacun, tout en reconnaissant au groupe une capacité à dépasser la seule somme des initiatives personnelles. Travailler de manière autonome exige également d'identifier les styles 
cognitifs de chaque apprenant (Rogers, 1968), en s'appuyant sur la force de chacun, tout en travaillant les points faibles afin de les améliorer dans un contrat enseignant négocié et accepté de chacun.

\section{Le collectif dans l'autonomie}

9 Trop longtemps l'apprentissage des savoirs fondamentaux, comme les approches plus récentes centrées sur les compétences, se sont focalisées sur l'individu apprenant, en omettant de considérer la place et le poids du collectif dans les acquisitions de savoir. Proche de la pédagogie Freinet (Peyronie, 2013 ; Capelle, Lehmans, Liquète, 2017), le postulat des FabLabs est que l'on s'enrichit personnellement à partir d'une organisation du travail et de la planification des tâches envisagées dès le départ sur la base d'un collectif d'individus. Dans ce collectif, chacun a besoin d'information pour avancer mais aussi besoin de communiquer, et les apprentissages se construisent largement dans la dynamique de communication. La posture autonome attendue revient alors à être en mesure de se positionner dans l'activité collective, d'en sortir pour réaliser d'autres tâches au fur et à mesure et d'auto-évaluer son apport et les éléments manquants pour que l'apprentissage soit complet. C'est également une posture autoréflexive qui est attendue de la part des enseignants et des médiateurs impliqués (Morandi, 2001).

\section{La motivation et la persévérance}

10 Enfin, le FabLab, comme la manipulation de robots, permettent de travailler sur une dimension essentielle pour les apprentissages et la persévérance, celle de la motivation. Le rôle et l'importance de la motivation dite "intrinsèque " " ont été le sujet de plusieurs études dès les années 1950 en psychologie et en pédagogie. La motivation intrinsèque s'oppose à la motivation extrinsèque dans la mesure où son objet n'est pas la satisfaction de besoins liés à des stimuli extérieurs spécifiques, comme la réponse aux besoins primaires, mais l'attrait de certaines activités pour « elles-mêmes » et ce qu'elles représentent pour l'apprenant. Les comportements que l'on attribue d'ordinaire à la curiosité, à la recherche de la nouveauté ou au plaisir de l'exploration, seraient le résultat de ce type de motivation. La relation entre la motivation intrinsèque et l'efficacité des apprentissages à l'école a été montrée de nombreuses fois dans la littérature, et plusieurs méthodes ont été ainsi développées pour la mesurer expérimentalement, notamment sur la base de questionnaires (Ryan, Deci, 2000). Ainsi, persévérance scolaire et motivation dans l'acquisition de savoirs et de compétences sont étroitement liées, la démarche par essai-erreur de l'apprenant ayant toute sa place.

11 Célestin Freinet a été l'un des premiers à proposer d'ouvrir l'école sur le monde extérieur et ses réalités concrètes, comme dans le cas du jardin pédagogique. Dans le système scolaire actuel, en effet, les élèves en décrochage scolaire, ayant perdu toute appétence pour l'apprentissage, inadaptés à une organisation scolaire rigide réglée par l'évaluation et la sanction, sont susceptibles de retrouver le plaisir d'apprendre dans des "outre-lieux" tels que les Fablabs, qui permettent de passer de la conception au prototypage puis de la mise au point à la réalisation d'objets. Ces lieux d'apprentissage et espaces de créativité favorisent la rencontre et les interactions de différents acteurs autour d'objets partagés pour des usages différenciés dont on fait l'hypothèse qu'ils peuvent aider des jeunes en difficulté ou en décrochage par rapport à leur parcours 
scolaire. En effet, les compétences dans l'activité valorisent l'estime de soi à travers un espace-temps en décalage par rapport à l'école, qui laisse toute sa place à la démarche d'essai-erreur et aux interactions. Le travail accompagné se réalise le plus souvent à plusieurs, sur le mode de projet visant à s'organiser pour atteindre un objectif commun. L'atteinte de cet objectif représente alors une réussite qui permet de regagner confiance et de se sentir valorisé.

12 Dans ce cadre, les objectifs du projet visent une activité non maîtrisée (non formalisée à l'avance dans le détail des activités), nécessitant une réflexion, une adaptation, un entraînement (avec des échecs possibles dans une démarche d'essai-erreur) pour développer des tâches plus ou moins complexes. L'expérimentation repose sur l'hypothèse que la fabrication d'objets donne un sens à l'activité et aux apprentissages, crée des liens entre différents apprentissages et acteurs de l'éducation, suscite la curiosité et donc la motivation, valorise la réalisation et les compétences acquises.

\section{Problématique et éléments de méthode}

\section{Une problématique à trois niveaux}

13 Dans le cadre de la recherche et des démarches-actions engagées au sein du collectif Persévérons (recherche e-Fran 2016-2020) composé de chercheurs et d'acteurs de la médiation, nous avons choisi de réfléchir à une problématique de recherche imbriquant trois niveaux de réflexion :

14 - premièrement, le niveau individuel : dans quelle mesure, la robotique éducative engagée dans les FabLabs permet-elle d'augmenter les niveaux de persévérance et de performance des apprenants observés par nos soins?

15 - deuxièmement, le niveau collectif : en quoi, le vivre et le construire ensemble, autour de temps de production et de réalisation négociés, permettent-ils à chacun de mieux vivre l'expérience pédagogique et d'augmenter le niveau de performance de chacun? En quoi le robot au centre de l'activité permet-il de formaliser le travail préparatoire des élèves?

16 - troisièmement, le niveau expérientiel : dans quelle mesure, l'apprentissage hors les murs de la salle de cours, dans un espace d'expériences et une approche de «médiation sensible » (Fabre, 2017) permet-il de mieux évoluer, d'oser et de prendre plaisir à vivre ensemble afin de réaliser au mieux un ensemble de tâches?

\section{Un recueil de données tridimensionnelles}

$17 \mathrm{Au}$ fur et à mesure de la construction d'un protocole revendiquant une appréhension complexe du micro-pédagogique, nous avons opté pour la triangulation des méthodes, combinant trois approches :

18 - d'une part, la description des dispositifs pédagogiques proposés par les enseignants et les médiateurs en tentant de caractériser les composantes clefs des agencements et des scénarii d'usages envisagés en amont de l'activité elle-même ;

19 - d'autre part, l'observation des activités en situation, en se centrant prioritairement sur les interactions et résolutions des tâches de la part des apprenants, tout en considérant les éventuels décrochages au cours des activités ; 
20 - enfin, le recueil de la parole des apprenants en fin de la séquence de travail afin de revenir sur les temps forts vécus et les moments clefs perçus par l'enquêteur, notamment afin de considérer les éléments d'explication et de rationalité portés par les acteurs; ceci nous permet de recueillir des verbatim en tant qu'unités d'explication au sens de la réalité et de la rationalité de l'apprenant.

Dans ce recueil, plusieurs dimensions sont particulièrement visées :

22 - les modes de travail et de communication dans le cours du projet, la spatialisation, la temporalité ;

23 - les interactions sociales : dans une journée, sur un temps plus long (entre les élèves, entre les adultes et les élèves), les affiliations ou désaffiliations ;

24 - les interactions hommes-machines, les évolutions des comportements et des modes de travail, les méthodes de recherche, de recueil d'information, de projection et de fabrication, de communication autour des projets des élèves.

\section{Le contexte malien}

\section{La robotique au Mali}

25 La robotique, bien qu'elle constitue une nouveauté et qu'elle ne soit pas encore vraiment ancrée dans les pratiques technologiques africaines, commence à gagner du terrain sur le continent. Des travaux qui font appel à la robotique sont répertoriés un peu partout en Afrique. Au Sénégal la première édition de la Panafrican Robotics Competition (PARC) a eu lieu en 2016. Au Togo, un jeune s'est lancé dans la transformation des déchets électroniques en robots. Il parcourt les écoles avec son robot pour sensibiliser les élèves à l'importance de la protection de l'environnement à travers le recyclage et les intéresser aux technologies numériques. Dans le domaine de l'éducation, des centres et des stages de formation, des écoles en robotique ont vu le jour au Cameroun, en Namibie, au Rwanda et en Afrique du Sud en 2018. Certaines de ces écoles dépassent le cadre éducatif pour tendre vers un cadre sociétal. Elles proposent des programmes encourageant les filles dans la robotique. C'est le cas du centre NextGen au Cameroun et du centre de formation de la Meta Economic Development Organization en Afrique du Sud. Elles sont destinées à contribuer à l'émancipation des femmes, considérée comme un défi majeur à relever pour l'Afrique. En 2018, le Mali a inauguré son premier centre de formation en robotique. L'ouverture de ce centre a été motivée par le succès en 2017 d'un groupe de jeunes lycéens et collégiens au Panafrican Robotics Competition (PARC) et au concours de robotique organisé par la First Global à Washington. Les jeunes maliens ont remporté le deuxième prix sur 157 équipes. Ce groupe d'élèves se compose de 20 lycéens et 21 collégiens. Ils ont été formés par l'Education pour un Monde Moderne Mali Emergent Robots Mali (EMMME) en collaboration avec le Ministère de l'Enseignement Supérieur et de la Recherche Scientifique (MESRS) du Mali suite aux propositions formulées lors de la première édition de la Fête des Sciences en décembre 2016. L'objectif de cette rencontre est d'offrir un espace d'échange et de réflexion pour attirer le plus d'élèves vers les filières scientifiques et techniques.

Avec l'appui de L'UNESCO en 2018, ces élèves ont suivi un ensemble d'activités visant à acquérir des compétences en robotique et à vulgariser la robotique dans le monde éducatif. Ces activités étaient, entre autres, centrées sur la formation d'une équipe 
nationale de robotique pour participer aux compétitions internationales, la création de clubs de robotique dans les établissements scolaires et universitaires, l'ouverture d'un espace universitaire de développement des projets robotiques. En 2018, l'équipe participe à la deuxième édition de la Panafrican Robotitcs Competition au Sénegal. D'autres pays africains comme le Nigéria, le Sénégal et l'Afrique du Sud ont également participé à cette compétition. L'équipe malienne a remporté le premier prix de la catégorie TECH League et ENGINEERS League et le troisième prix de la catégorie STAR league. Ces activités ont motivé plusieurs jeunes qui ont investi dans des start-up, comme Donilab, qui, en plus de proposer d'autres services, offre un espace de collaboration autour du numérique et de la robotique. D'autres passionnés de robotique et n'ayant ni accès aux espaces dédiés ni soutien des institutions s'organisent tant bien que mal pour échanger leurs connaissances. C'est le cas des étudiants de l'Université de Ségou, à travers la création d'un FabLab.

\section{L'émergence de FabLabs africains}

Depuis quelque année nous assistons à une émergence considérable du nombre d'espaces de partage, d'échange, de collaboration et de création numériques en Afrique. Une aubaine qui permet de réduire la fracture numérique et de répondre à des enjeux environnementaux et éducatifs. À partir des opportunités et de l'évolution du numérique, des Fablabs ont été créés un peu partout. En 2018 le Réseau francophone des Fablabs d'Afrique de l'Ouest (ReFFAO) est lancé au Benin. Il regroupe une dizaine de Fablabs du Benin, Togo, Burkina Faso, Côte d'Ivoire, Mauritanie, Sénégal et du Mali. Les objectifs de la création de ces Fablabs diffèrent: offrir un espace d'innovation ouvert, comme celui du Woelab; préserver et ltransmettre des savoir-faire traditionnels, comme celui de Studio Wudé ; répondre aux besoins d'une communauté, comme le cas de DefKo Ak Niep; ou créer un appui pédagogique. Le Polylab de l'Université Thiès entre dans ce cadre. Selon Stéphanie Leyronas et Tamatoa Bambridge (2018), les fablabs Africain se distinguent de leurs homologues européens et américains par trois traits.

- L'innovation frugale et la "bidouille" sont au cœur du dispositif. Ils se proposent de trouver une solution aux problématiques locales avec le peu de ressource à leur disposition privilégiant le recyclage et les outils open source.

29 - Ils s'inscrivent dans des contextes économiques et financiers difficiles. La majorité sont ouverts sur les seuls fonds propres de leur fabmanager. Certains bénéficient de l'appui financier d'un organisme international comme la Fondation Orange Solidaire ou OIF. Ces appuis sont aléatoires et ne les sécurisent pas financièrement. La plupart proposent des services payants pour la survie de l'espace.

30 - Ils sont producteurs à la fois de communs informationnels mais aussi de communs éducationnels. Ils proposent des ateliers de formation à destination d'un public large : le Jerry school pour OuagaLab (Burkina Faso), un programme pour l'apprentissage d'assemblage d'ordinateurs à destination des élèves du primaire; des camps de formation pour BabyLab (Côte d'Ivoire) et Defko (Sénégal); la formation des professionnels locaux pour Blolab (Bénin), Ker Thiossane (Sénégal).

\section{Première démarches d'expérimentation : I'université de Ségou}

Dans le cadre de notre projet de recherche, une étude a été menée pour analyser l'effet d'un Fablab dans l'accompagnement pédagogique des étudiants dans le contexte 
malien. D'une part, elle a consisté à la mise en place d'un Fablab à l'Université de Ségou, d'autre part, à mettre des étudiants en activité sur des projets autour de la robotique dans ce lieu. L'observation participative et les entretiens ont permis de recueillir des données.

Le Fablab mis en place, SégouLab, dispose de deux espaces : une salle technique et un espace d'échange et de restitution. La salle technique contient des outils numériques et des kits robotiques. Elle est ouverte aux étudiants et enseignants pour la réalisation des projets pédagogiques. L'espace d'échanges et de restitution appelé «CHIKORO » (sous l'arbre de karité) est un espace en plein-air dans lequel sont organisées les séances de restitution des travaux où chacun expose son projet, l'état d'avancement de celui-ci et les points de blocage. Chaque projet est examiné par les équipes participantes. Des pistes de solutions sont proposées et débattues. Cette forme d'organisation est motivée par le souci de prendre en compte une pratique communicationnelle répandue au Mali. Cette pratique consiste à se réunir pendant les heures de pause, généralement par tranche d'âge, en plein-air, pour partager du thé et discuter de l'actualité. Ces lieux sont des espaces dans lesquels les informations et les connaissances sont mises "en commun", des communs de la connaissance.

Les étudiants accueillis, au nombre de dix-neuf, six filles et treize garçons dont six professionnels, ont été répartis en quatre groupes. Ils ont été initiés à la programmation et ont échangé avec les stagiaires et accompagnateurs du Fablab Coh@bit ${ }^{1}$ par Riot ${ }^{2}$, l'objectif étant de concevoir des robots.

\section{Premiers résultats : éléments de tendance recueillis}

\section{Apprendre autrement}

Il faut souligner que les étudiants n'avaient jamais visité physiquement un Fablab ni été en contact avec les outils numériques tels que les imprimantes 3D ou la découpeuse laser qu'on trouve dans les Fablabs. L'expérience s'est déroulée sur cinq jours (du lundi au vendredi). Le premier jour de découverte de la robotique s'est terminé avec une séance de restitution à CHIKORO qui a donné lieu à la rédaction d'un rapport journalier, comme tous les autres jours. Le deuxième jour, les apprenants ont travaillé sur l'impression 3D. À partir du troisième jour jusqu'au dernier, les étudiants ont travaillé sur la fabrication des robots avec les pièces mises à leur disposition.

À l'issue de cette expérience, il ressort que la motivation, l'autonomie et le développement personnel des étudiants sont influencés par la participation au projet. Les séances censées se terminer à 18 heures s'étiraient jusqu'à 20 heures. Les étudiants expliquent : «On a pensé que le temps était trop juste pour terminer alors qu'on voulait absolument voir le robot fonctionner ", «la nuit, la connexion internet est beaucoup plus stable, ça nous permet d'aller plus loin dans nos recherches » ou encore «j'avais dit à la maison qu'on fabriquait un robot, ils ne me croyaient pas. Il fallait que ce robot marche ». Ces propos dénotent leur engagement dans le projet. Cet engagement était si fort qu'ils ont transformé des tâches scolaires en projets personnels, ce qui peut s'expliquer par leur perception de l'environnement du Fablab. Ce dernier met à leur disposition un espace d'échange en harmonie avec leurs pratiques d'information et de communication, des outils numériques nouveaux pour eux et des pièces électroniques hors de leur portée dans le contexte universitaire "normal ". Ils étaient motivés. 

prendre en charge son apprentissage. C'est tout un processus qui se construit dans un collectif pour amener l'apprenant à mobiliser ces connaissances antérieures, interagir et s'adapter pour pouvoir construire son propre savoir (Liquète, Maury, 2007). Elle sous-entend que l'apprenant, dans ce processus, collabore avec ses pairs et soit orienté, encouragé par l'enseignant. L'enseignant doit passer du rôle de prescripteur à celui d'accompagnateur pour que l'apprenant puisse s'autoévaluer et évoluer par essaiserreur. Les étudiants sont parvenus à une organisation centrée sur la communication et la collaboration dans laquelle il était difficile de distinguer les différents groupes. Dans un groupe, un étudiant (professionnel) affirme : «on dit souvent lorsqu'on veut aller vite il faut aller seul mais lorsqu'on veut aller plus loin, il faut aller avec les autres ». Tous affirment avoir collaboré avec les autres, membres de leur groupe ou d'autres groupes. Ils affirment aussi avoir apprécié la posture de l'enseignant. Celui-ci avait adopté une posture d'accompagnateur. Selon une étudiante "Je sais qu'on est venu à l'école pour travailler mais de temps en temps ne pas faire de différence entre le professeur et l'élève, je pense que ça doit être considéré. Ça met l'élève à l'aise, ça lui donne le courage de travailler ». Un autre étudiant (professionnel) nous dit d'avoir été « émerveillé » par «le climat entre professeur et étudiants " parce que «quelque part on ne pouvait pas reconnaître qui est le professeur qui est l'étudiant ». Fab semble ainsi favoriser le développement personnel qui vise une «transformation de soi ", soit pour se "défaire de certains aspects pathologiques" comme la phobie, l'anxiété, la timidité ou pour améliorer ses performances (Wagnon, 2018). Une étudiante (régulière) nous fait savoir qu'elle a amélioré ses performances tant sur le plan communicationnel que sur le plan rédactionnel. Le travail au Fablab a libéré ses capacités d'écriture qu'elle ne soupçonnait pas. Elle nous dit: " personnellement si on avait dit de faire un rapport sur tout ce qu'on a vu en classe, je n'allais pas dépasser au maximum deux pages mais là je parviens à dépasser au minimum deux pages par jour ». D'autres nous rapportent qu'ils avaient des difficultés à s'exprimer ou à travailler en groupe et que la semaine leur a permis de surmonter ces difficultés. Ainsi, "travailler en équipe ou en groupe, j'avais des difficultés, ça m'a permis de surmonter ces difficultés et je pense que, dans les jours à venir, le travail en équipe ne me causera plus de problème ». Une étudiante (régulière) peut s'exprimer paisiblement, alors que «avant la semaine, je m'énervais quand j'expliquais et que les autres ne me comprenaient pas ».

Il semble donc que le projet sur la robotique et les dispositifs numériques, tous deux nouveaux pour ces étudiants, conjugués à l'espace, sont à la base des impacts observés. Fabriquer des robots en utilisant les dispositifs numériques nouveaux dans un espace culturellement familier est à l'origine de la persévérance des étudiants. Cette expérience met en lumière l'importance d'un Fablab dans l'accompagnement pédagogique des étudiants à Ségou. Elle soulève aussi la question de la difficulté de la mise en place et la pérennité d'un tel lieu. Cette difficulté est d'ordre matériel et humain pour l'accompagnement des étudiants. Les dispositifs numériques qu'on retrouve dans les Fablabs sont rarement disponibles sur le marché malien. Peu de sites de vente en ligne livrent au Mali. Ces facteurs rendent les conditions d'acquisition difficiles et augmentent considérablement les frais de matériels indispensables dans les Fablabs. L'utilisation pose également problème, étant donné qu'il y'a peu d'enseignants qui maîtrisent ces outils et sont disponibles pour accompagner les étudiants.

Communication, technologies et développement, 8 | 2020 


\section{Les conditions d'un essaimage ouvert à l'interculturel des apprenants et des enseignants}

Les Fablabs sont des espaces privilégiant la collaboration et le partage de savoirs et de savoir-faire. Ces collaborations et partages au départ internes, pour prendre en charge les besoins d'une communauté donnée, se sont rapidement externalisés pour atteindre une dimension beaucoup plus large. Cela peut aller du partage des techniques de fabrication sur des plate-formes libres d'accès à l'essaimage en vue de créer des sousespaces. Le site thingiverse ${ }^{3}$ est une plate-forme de partage orienté impression 3D. Des milliers de model 3D, directement imprimables sont accessibles gratuitement. Il offre la possibilité de partager ses models 3D avec les autres. Le wikilab ${ }^{4}$ est une sorte d'encyclopédie où tout «maker » peut partager ses tutoriels en accès libre et gratuit. Les tutoriels sont détaillés à tel point qu'ils précisent le niveau de difficulté, la durée et le coût pour chaque objet en question. En plus du français, les tutoriels sont en anglais, espagnol, italien, portugais et/ou en allemand. Le site propose même après avoir créé un compte de participer à la traduction de la plateforme dans d'autres langues. Il est clair que cette plate-forme met un accent particulier sur l'interculturalité et tente de l'intégrer dans son processus de démocratisation du savoir. Le partage de l'information et de la documentation sont à la base de ce processus, au Mali comme en France, et entre les pays. On voit se construire dans les Fablabs une véritable culture du partage autour des données, que les élèves et les étudiants expérimentent personnellement.

La dynamique de l'essaimage est à la base de l'expansion des Fablabs dans le monde. Elle est le résultat des rencontres inter-individuelles, de l'utilisation d'espaces et de la participation à des événements « makers ». L'association Ping ${ }^{5}$, par exemple, est à l'origine de la création du centre social du chemillois et du Baraka'Lab dans le pays de Loire. Deux makers bretons sont allés créer un makerspace à Hué au Vietnam en 2013. Doualab au Cameroun a vu le jour suite à la visite du promoteur du Fablab Electrolab de Nanterre ${ }^{6}$. Ce mouvement de Fablabs favorise l'interculturalité et pourrait permettre aux étudiants et enseignants de découvrir d'autres réalités. L'ouverture à l'interculturel des étudiants et enseignants peut se concrétiser à travers des échanges à l'image de projets en commun à distance de groupes d'étudiants de différentes universités, de stages dans des Fablabs des pays étrangers et/ou par des programmes de tutorat à distance.

\section{Conclusion}

41 L'ampleur des phénomènes de décrochage scolaire et des défis que rencontrent tous les pays, particulièrement en Afrique, pour assurer une scolarité à tous, est de plus en plus préoccupante. C'est du côté de l'école et de l'université que des actions peuvent être mises en place avec des perspectives de changements significatifs si le levier de la motivation est correctement activé. La robotique, comme le numérique plus largement, sont souvent considérés comme un élément déterminant de ce levier, pas tant à cause de la fascination pour l'objet technologique ou pour l'image, que dans leurs potentialités pédagogiques et les perspectives de renouvellement des pratiques de classe. Le Fablab démultiplie des effets de levier, tout en ouvrant les perspectives d'éducation et de formation vers une culture du partage, et pas seulement une culture 
des données. Que l'on parle de pédagogie du détour, du jeu, ou du projet, la robotique et les Fablabs permettent d'intégrer des formats et des modalités pluriels d'apprentissage, de renverser la perspective de l'enseignement frontal qui a marqué ses limites, tout en repensant la question de l'autonomie dans les apprentissages et du partage de la connaissance.

\section{BIBLIOGRAPHIE}

BACHIMONT, B. Computation. In Frau-Meigs, D., Kiyindou, A., Musitelli, J., La diversité culturelle à l'ère du numérique : glossaire critique, Paris, La Documentation française, 2015, p. 80.

BANDURA, A. 1977. Social Learning Theory. Prentice Hall, 1977.

BARBEAU, D. Les sources et les indicateurs de la motivation scolaire. dans Actes du 13e colloque de l'AQPC. Les collèges, une voie essentielle de développement, AQPC, 1993.

BAUDRILLARD, J., Le système des objets, Paris. Gallimard, 1968.

BOSQUE, C., RICARD, L. FabLabs, etc. Les nouveaux espaces de fabrication numérique. Paris, Eyrolles, 2015.

BRUILLARD, E. Lire-écrire-computer : émanciper les humains, contrôler les machines. Ina-Global. URL ; https://www.ina-expert.com/e-dossier-de-l-audiovisuel-l-education-aux-cultures-de-linformation/lire-ecrire-computer-emanciper-les-humains-controler-les-machines.html, 2012.

CAPELLE, C., LEHMANS, A., LIQUETE, V. Culture numérique partagée et modalités de coconception des apprentissages en régime numérique. Colloque international : coopération, formation, la pédagogie Freinet face aux défis du XXIème siècle, Jul 2017, Bordeaux, France.

DECI E. L., CASCIO W.F., Changes in intrinsic motivation as a function of negative feedback and threats, Eastern Psychological Association Convention, Boston, 1972.

DECI, E. et RYAN, R. The" what" and" why" of goal pursuits: Human needs and the selfdetermination of behavior. Psychological inquiry, 2000, vol. 11, no 4, p. 227-268.

DEWEY, J. Démocratie et éducation : suivi de Expérience et Éducation. Paris, Armand-Colin, 2011. DIDIER, J. et QUINCHE, F. Concevoir des robots pour développer la créativité des élèves ?. Voix Plurielles, 2016. vol. 13, no 1, p. 101-112.

FABRE I., Médiation du contemporain : expérience esthétique de dispositifs documentaires. Retour réflexif sur les pratiques professionnelles. Habilitation à diriger des recherches (HDR) en sciences de l'information et de la communication, Université Toulouse 2 Jean Jaurès, 2017.

FREINET, C. Oeuvres Pédagogiques, Tome 1. Paris : Seuil, 1994.

GOODY, J. La Raison graphique, Paris, Minuit, 1979.

KAPLAN F. et OUDEYER, P.-Y. Le corps comme variable expérimentale, Revue philosophique de la France et de l'étranger, 2008, Tome 133 - n 3, p. 287-298. 
LAVE, J., WENGER, E. Situated Learning: Legitimate Peripheral Participation. Cambridge University Press, 1991.

LEROI-GOURHAN, A. Le geste et la parole, tome I Technique et langage. Paris, Albin Michel, 1964.

LEYRONAS, S., BAMBRIDGE, T. Communs et développement : une approche renouvelée face aux défis mondiaux. Revue internationale des études du développement, 2018, 233-1, p. 11-29.

LHOSTE, É., BARBIER, M. FabLabs. L'institutionnalisation de Tiers-Lieux du « soft hacking » ", Revue d'anthropologie des connaissances, 2016, vol. 10, $\mathrm{n}^{\circ} 1$, p. 43-69.

LIQUÈTE V., MAURY Y., Le travail autonome : comment aider les élèves à l'acquisition de l'autonomie, Paris, Armand Colin, 2007.

MORANDI ,F. Modèles et méthodes en pédagogie, Paris, Nathan, 2001.

OSTROM, E. Gouverning the Commons. The Evolution of Institutions for Collective Action. Cambridge UP, 2015.

OUDEYER, P.-Y. Des ordinateurs aux robots : les machines en informatique, 2016. https:// hal.inria.fr/hal-01404432/document

PAPERT, S., Le jaillissement de l'esprit. Ordinateurs et apprentissages. Flammarion, Paris, 1981.

PEYRONIE H., Le mouvement Freinet : du fondateur charismatique à l'intellectuel collectif. Regards sociohistoriques sur une alternative éducative et pédagogique, Caen, Université de Basse-Normandie, Centre d'études et de recherches en sciences de l'éducation, 2007.

ROGERS C., Le développement de la personne, Paris, Dunod-Bordas, 1968.

ROY, D. « Optimisation des parcours d'apprentissage à l'aide des technologies numériques. Environnements Informatiques pour l'Apprentissage Humain ». Thèse, CNAM, Paris, 2015. https://tel.archives-ouvertes.fr/tel-01252695/document

SIMONDON,, G. "Entretien sur la mécanologie". Revue de synthèse, Tome 130, 6e série, nº 1, 2009, URL : https://monoskop.org/images/4/48/

Simondon_Gilbert_Le_Moyne_Jean_1968_2009_Entretien_sur_la_mecanologie.pdf

STALLMAN, R. Le libre comme alternative pour l'école contemporaine? ». Hermès, La Revue. vol. 78, no. 2, 2017, p. 104-107.

SUIRE, R. La performance des lieux de cocréation de connaissances. Réseaux, no 2, p. 81-109, 2016.

TISSERON, S. Le jour où mon robot m'aimera. Vers l'empathie artificielle. Paris, Albin-Michel, 2015.

TRICOT, A. L'innovation pédagogique. Paris, Retz, 2017.

VALLAT, D. Que peut-on apprendre des tiers-lieux 2.0 ?. XXVIe conférence de l'AIMS (Association Internationale de Management Stratégique), Jun 2017, Lyon, France. https://halshs.archivesouvertes.fr/halshs-01512929/document

WAGNON., S. Les pédagogies alternatives en France aujourd'hui : essai de cartographie et de définition. Tréma, 2018.

WING, J. M. 2006. Computational thinking. Communications ACM. vol. 49, $\mathrm{n}^{\circ}$ 3, mars 2006, p. 33-35.

\section{NOTES}

1. Le Fablb Coh@bit est un des partenaires du projet Perseverons et a accueilli Mohamed Coulibaly en stage durant l'année 2018 : https ://www.iut.u-bordeaux.fr/cohabit/ 
2. Outil permettant aux usagers d'internet de passer des appels téléphonique ou video : https :// about.riot.im

3. https ://www.thingiverse.com

4. https ://wikifab.org

5. https ://info.pingbase.net

6. http://www.makery.info/2017/04/28/tribulations-dun-maker-en-afrique-longola-fablabouvre-a-yaounde/

\section{RÉSUMÉS}

Depuis quelques années, de nouveaux espaces d'apprentissage et de production ont vu le jour sous forme de FabLabs. Cet article se propose de présenter et d'analyser l'intérêt de la robotique éducative au sein de ces nouveaux d'espaces, tout en montrant les conditions et les difficultés à transférer ces espaces et ces pratiques dans plusieurs contextes, à travers une première initiative au Mali.

Recently, FabLabs have been used and considered as new learning and production spaces. This communication will present and analyze activities in the field of educational robotics within these new spaces, while showing the interest, conditions and limits to transfer these spaces and practices in other contexts, like in an initiative in Mali.

En los últimos años, han surgido nuevos espacios de aprendizaje y producción en forma de "FabLabs". Este artículo propone presentar y analizar actividades en el campo de la robótica educativa dentro de estos nuevos espacios, al tiempo que muestra las condiciones y dificultades para transferir estos espacios y prácticas en otros contextos, a través de una primera iniciativa en Mali.

\section{INDEX}

Mots-clés : fabLab, espace collaboratif, robotique éducative, apprentissage collectif, interculturalité, commun de la connaissance

Palabras claves : Espacio colaborativo, Robótica educativa, Aprendizaje colectivo, Interculturalidad, Conocimiento común

Keywords : FabLab, Collaborative Space, Educational Robotics, Collective Learning, Interculturality, Knowledge Commons

\section{AUTEURS}

\section{ANNE LEHMANS}

Maître de conférences en Sciences de l'information et de la communication, Université de Bordeaux, IMS-UMR5218

anne.lehmans@u-bordeaux.fr 


\section{VINCENT LIQUÈTE}

Professeur des université en Sciences de l'information et de la communication, Université de Bordeaux, IMS-UMR5218

vincent.liquete@u-bordeaux.fr

\section{MOHAMED COULIBALY}

Université de Ségou, Mali, Université de Bordeaux

mohamed.coul.info@gmail.com 